\title{
FORMULACIÓN DE LA CADENA PRODUCTIVA DEL CARACOL EN CUNDINAMARCA Y BOYACÁ - COLOMBIA PARA EXPORTAR HACIA EL MERCADO ESPAÑOL
}

\section{FORMULATION OF THE SNAIL PRODUCTIVE CHAIN IN THE DEPARTMENTS OF CUNDINAMARCA AND BOYACA - COLOMBIA FOR EXPORT TO THE SPANISH MARKET}

\author{
Álvaro Lamprea Delgado ${ }^{1}$ \\ María Concepción Salazar Alonso² \\ Adriana Posada Arrubla
}

\section{RESUMEN}

Para formular la cadena productiva del caracol, se consideraron dos referentes: la Unión Europea, específicamente España, y la normatividad en Colombia, en aspectos relacionados con la producción y la comercialización. Se propuso la cadena para desarrollar una oferta exportable de productos de uso alimenticio, para generar empleo, mejorar los ingresos para pequeños y medianos productores y consecución de divisas, permitiendo potencializar esta actividad agroindustrial, recién creada en Colombia. Esta investigación permitió determinar las oportunidades que tiene la exportación de caracol en el mercado español, lo mismo la actividad helicícola en Colombia, teniendo en cuenta los aspectos más relevantes de la cadena, como las empresas, los gremios, entre ellos Fedecohel, las universidades, el

${ }^{1}$ Profesional en Comercio Internacional, Especialista en Gerencia de Mercadeo, Docente Investigador U.D.C.A. Dirección para correspondencia: alamprea@udca.edu. $\mathrm{CO}$

${ }^{2}$ Economista, Docente Investigadora U.D.C.A. Dirección para correspondencia: masalazar@udca.edu.co

${ }^{3}$ Economista Agrícola, Especialista, Magíster. Dirección para correspondencia: aposada@udca.edu.co sector financiero, además del gobierno. Esto para que se realicen gestiones coordinadas y lograr, en el futuro, proveer, en forma garantizada, un suministro de productos procesados o derivados de la carne del caracol y los subproductos, que se puedan producir y comercializar en economías a escala, en términos de volúmenes de producción, de calidad, de logística de distribución física, a nivel nacional e internacional, de empaques y embalajes y términos de negociación en el mercado meta, que permitan cumplir con los pedidos en el exterior. Para tal fin, se debe transformar el sistema tradicional de producción, para que sea más productivo y competitivo y, de esta forma, permitir a las empresas nacientes competir y acceder a los mercados internacionales, especialmente, al español.

Palabras clave: Helix aspersa, cadena productiva, comercialización internacional, producción, exportaciones.

\section{SUMMARY}

To formulate the snail productive chain two referents, the European Union one, specifically, Spain, as well as the Colombian norms, regarding aspects as production and commercialization, were considered. The chain, to develop an offer of exportable products of alimentary use, to generate employment, income improvement for 
small and medium producers, as well as the achievement of devises, allowing to potentialize this agro-industrial activity, recently created in Colombia, is proposed. This research allowed to determine the opportunities of the snail export to the Spanish market and the Helicicola activity, having in mind the most relevant aspects of the chain, the companies, the associations as Fedecohel, the universities, the financial sector and furthermore the government. This, in order to develop coordinated activities and in the future, to supply, in a guaranteed manner, processed snail products or meat derived and sub-products, produced and commercialized in economies, at scale, in terms of production, volume, quality, logistic of physical distribution at national and international level, paking and terms of negotiation in the market that allow them to fulfil the international market. For this, the traditional system of production has to be transformed, to make it more productive and competitive and this way to allow the nascent companies to compete and access the international markets, especially the Spanish one.

Key words: Helix aspersa, productive chain, international commercialization, production, export.

\section{INTRODUCCIÓN}

El caracol comestible, Helix aspersa, introducido a Colombia en el siglo pasado desde Brasil (Rodrigues, 1991), es una fuente alimenticia muy importante y un mercado en potencia, desaprovechado en el país, por no existir la cultura interna de su consumo, ni existe una cadena de valor que permita la comercialización adecuada, que cubra la demanda insatisfecha de los mercados internacionales.

En el ámbito internacional, los mayores consumidores de carne de caracol son Francia, España e Italia, donde España, se destaca por ser un importante consumidor y productor de caracoles, tradición que no solo se da a nivel de restaurantes sino también en los hogares, siendo la mayor demanda en otoño y durante las fiestas de navidad y fin de año. Es importante destacar cómo la ventaja que supone producir en contra de la estación de invierno es invalorable y pone a Colombia en situación de privilegio (Giraldo et al. 2006). España Importa alrededor de 4.000t anuales de caracol (Corporación Financiera Nacional CFN, 2003); durante temporada alta, se ofrece a 2,50€/kilo caracol vivo y en temporada baja supera los $4 €$, llegando hasta $5 €$, el caracol precocido.

A partir de estas referencias, se infiere que España es demandante y Colombia es un potencial oferente, debido a que presenta una ventaja competitiva por las condiciones climáticas, que permiten una producción a gran escala durante todo el año, mientras que España, por sus estaciones, solo puede producirlos, a gran escala, en verano. Por lo cual, puede ofrecer el caracol a unos precios competitivos para exportación que oscilan entre $1,88 €$ y $3,40 €$, lo que representa un margen de rentabilidad 0,62€ por kilo, para el empresario (García, 2005). Sin embargo, la helicicultura en Colombia es una agroindustria naciente, que requiere de procesos productivos y comerciales tecnificados, para poder desarrollar una oferta exportable.

En América Latina, especialmente, Argentina, Perú y Chile, son los principales países exportadores de caracol al viejo continente, abriendo posibilidades para Colombia, debido a la alta demanda internacional, estimada en 300 mil toneladas año. Pero precisamente para poder ingresar al mercado europeo, se deben cumplir con ciertos requisitos, como el certificado sanitario, seriedad, calidad y compromiso de continuidad, aspectos que ya poseen los países europeos (Giraldo et al. 2006) y que en Colombia, se deben mejorar.

En el esfuerzo de Colombia para reproducir el caracol en cautiverio, se han introducido algunas especies, invasoras del medio natural por el manejo inadecuado que se le ha dado a los cultivos, por el abandono de los zoocriaderos y por las malas prácticas de manejo, lo que causa una dispersión descontrolada en algunas regiones del país, por lo cual, en algunos casos, se considera como una plaga o invasor (REUNA, 2005). Por ello y con el fin de proteger el ambiente, el Ministerio de Ambiente Vivienda y Desarrollo Territorial no seguirá autorizando nuevas solicitudes de licencias ambientales de introducción de la especie $H$. aspersa, de conformidad con lo dispuesto en el numeral 6, del artículo 1, de la Ley 99 de 1993 y en el literal h, del artículo 8, de la Ley 165 de 1994, inmerso en el Convenio Sobre la Diversidad Biológica.

La forma más efectiva de controlar el mal manejo de los caracoles y reducir su impacto ambiental es permitir y fomentar su comercialización y el aprovechamiento de sus productos y subproductos, que podrían ser una 
oportunidad y no una amenaza para desarrollar un nuevo subsector de la agroindustria colombiana. En la medida que se produzcan comercialmente, se reduciría el impacto ambiental, por que las autoridades ambientales podrían ejercer un efectivo control sobre esta actividad (Rousselet, 1986).

Por lo anterior, es importante determinar el tipo de aprovechamiento y seleccionar aquellas producciones que protejan el ambiente y generen una actividad económica sostenible y sustentable, acorde con la reglamentación nacional e internacional. Existen, por ejemplo, diferentes formas de producción para el caracol de tierra, una de ellas, identificar cuál se adecua más a las necesidades de los pequeños y medianos cultivadores en Colombia, por lo cual, se hace necesario tener el conocimiento y la tecnología que requiere este tipo de cultivos.

Un aspecto de referencia para programar el mercado colombiano de caracoles hacia el español, es que España, que ocupa el segundo lugar como demandante, después de Francia, cuenta con centros logísticos, importantes puertos, como Valencia, puerto atractivo por ser uno de los más eficientes, en el ámbito internacional, representando un punto estratégico para la distribución de alimentos hacia el resto del país (Montes E Montes, 2002). Pero a pesar de estas ventajas en infraestructura, no existe una normativa española específica para la producción, cría y comercialización del caracol, pues se regula por las normas generales de Sanidad y, la exportación, por los certificados exigidos en la aduana para la exportación del caracol vivo a España, como CONCAL (control de calidad), SANIM (Inspección sanitaria de importación), TSANR (Inspección sanitaria para el tránsito de mercaderías), VETER (Inspección veterinaria), CITES (Convención de Washington) (Sánchez, 2004).

\section{MATERIALES Y MÉTODOS}

El proceso investigativo desarrolló las bases para la comercialización de carne de caracol desde Colombia y hacia el mercado español en tres etapas, siendo una investigación aplicada de tipo cuantitativa y cualitativa, con las siguientes actividades por etapa:

1. En la primera etapa, se realizó la revisión documental sobre la helicicultura a nivel nacional e internacional, especificando a cerca de las condiciones del mercado y de la normatividad característica en Colombia, la Unión Europea y, particularmente, en España.

2. Conjuntamente con la Federación Colombiana de Helicicultores, se llevaron a cabo visitas de campo, entrevistas, la aplicación de la encuesta (Cuadro 1) y se realizó un focus group. En la encuesta, se analizan cinco variables, como se observa en el anexo (ambiental, sanitario, bioseguridad, productividad y comercialización), que fueron aplicadas a siete zoocriaderos seleccionados, aleatoriamente, de acuerdo a su sistema de cría, de producción y de medidas de manejo ambiental y comercial. En el focus group se incluyeron a 30 empresarios del sector, teniendo en cuenta la base de datos suministrada por Fedecohel y, para obtener la muestra, se aplico la fórmula para poblaciones finitas:

$\mathrm{n}=$ tamaño de la muestra ajustada $\mathrm{N}=$ población en este caso helicicultores

$$
\begin{aligned}
& \mathrm{n}^{`}=\text { muestra sin ajustar }=\mathrm{S}^{2} / \mathrm{V}^{2} \\
& \mathrm{n}=\frac{\mathrm{n}}{1+\mathrm{n}^{`} / \mathrm{N}}
\end{aligned}
$$

3. Se realizó, además, un sondeo directamente en la feria Alimentaría 2008, en Barcelona (España), que permitió evidenciar cómo en los mercados los españoles son posibles compradores, de los oferentes colombianos.

4. Por último, se llevó a cabo el análisis normativo de la ley 1011, de 23 de enero de 2006, del decreto reglamentario 2490, de julio de 2008 (por el cual se permite la producción y la comercialización helicícola en Colombia), de la Ley 165 de 1994 (que aprobó el Convenio Sobre la Diversidad Biológica), del numeral 6, del artículo 1, de la Ley 99 de 1993, del literal h, del artículo 8 del Ministerio de Ambiente Vivienda y Desarrollo Territorial, de la Ley 2490 del 2008 (sobre los requisititos sanitarios que deben cumplir los establecimiento dedicados al procesamiento, envase, transporte, expendio importación, exportación y comercialización de caracol con destino al consumo humano), todo ello, con el fin de determinar las posibilidades del mercado internacional de caracoles. 
Cuadro 1: Encuesta aplicada al grupo objetivo.

\section{UNIVERSIDAD DE CIENCIAS APLICADAS Y AMBIENTALES U.D.C.A \\ FACULTAD DE INGENIERÍA COMERCIAL}

Estudio producción y comercialización de la carne de caracol con destino al mercado español

\section{INFORMACIÓN GENERAL}

1.1 Representante Legal

1.2 Razón Social NIT

1.3 Dirección 1.4 Teléfono

1.5 Celular $1.6 \mathrm{E}$ - mail

\section{INFORMACIÓN PRELIMINAR}

Marque con una $X$

2.1 Tipo de sistema: Intensivo Mixto Extensivo Intensivo - Industrial

2.2 Especie de Caracol en Crianza Helix aspersa Müller Helix aspersa máxima

2.3 Tipo de Manejo Convencional Orgánico Mixto

2.3 Producción estimada en pie $\mathrm{kg}$, t, promedio de los últimos seis meses

\section{ASPECTOS DE COMERCIALIZACIÓN}

3.1 Tipo de comercialización Nacional Internacional

3.2 Canal de distribución Mayoristas Minoristas Otro Cual

3.4 Logística de la distribución (transporte) Terrestre Marítimo Aéreo

\section{ASPECTOS ADMINISTRATIVOS Y AMBIENTALES}

4.1 ¿Cuenta con licencia ambiental? Si No En trámite

4.2 Tipo de organización a que pertenece: Asociación ¿Cuál? Federación Cooperativa Otra

\section{RESULTADOS Y DISCUSIÓN}

La cría de caracol en cautiverio, con fines de exportación a España, es una oportunidad de negocio para los agricultores colombianos que buscan una alternativa de solución a los problemas que ha enfrentado el sector, debido a la apertura económica implementada en el país Lo anterior, aunado al tema ambiental que centra la atención en la conservación de las especies nativas enfrentado con la sostenibilidad de la producción de especies foráneas, como es el caso del caracol de tierra, caracterizado por tener un alto contenido proteínico y por permitir generación de ingresos a los helicicultores. Es de anotar que la Ley 811 de 2000, organiza esta actividad agroindustrial de forma tal, que posibilita desarrollar cultivos en ciclos de tipo abierto. 
En Colombia, la actividad helicícola es relativamente nueva, aunque existen agricultores que han realizado algunos desarrollos, que se encuentran en etapa incipiente. Hay diferentes razones que han llevado a los helicicultures a abandonar o a suspender la producción por diferentes factores, entre los que se destaca la falta de alianzas estratégicas de los helicicultores, que no les permite iniciar las exportaciones, para cubrir la demanda internacional.

Según FEDECOHEL, la región de la Sabana Cundiboyacense ofrece las mejores ventajas comparativas (clima estable, temperatura ideal, especie existente en forma silvestre) y cuenta con la logística para la comercialización internacional de los productos helicícolas. Adicionalmente, algunas entidades, como el SENA (Servicio Nacional de Aprendizaje), PROEXPORT (Fondo de Promoción de Exportaciones de Colombia) y la CCB (Cámara de Comercio de Bogotá), cuentan con programas para apoyar la creación y desarrollo de las pymes, oportunidades que podría ser aprovechada por los empresarios helicícolas.

Pero es importante también que el Ministerio del Medio Ambiente Vivienda y Desarrollo Territorial confiera a la helicicultura el estatus de especie promisoria enmarcado en el desarrollo sostenible y tenga viabilidad por parte de las entidades gubernamentales, con el propósito de crear y desarrollar una cadena productiva en un subsector nuevo (García, 2005) y que, en particular, se definan las condiciones de comercialización del caracol hacia el mercado Español.

La cadena productiva del caracol: Para desarrollar y mejorar la competitividad del sector helicícola en Colombia, se requiere diseñar y organizar una cadena productiva, que tenga en cuenta las necesidades de la demanda internacional y, a partir de esta, analizar a los actores y a los agentes participantes en el desarrollo de la misma. La cadena de valor es una alianza vertical o red estratégica entre un número de organizaciones empresariales independientes dentro de una cadena (Hobbs \& Cramer, 2000).

En el diseño de la cadena productiva, el enfoque de producción y de comercialización, es un punto de vista clave dentro del sector agroindustrial, debido a que identifica y analiza las barreras, las restricciones y/o puntos críticos de la cadena productiva del caracol, permitiendo un manejo de la información completo e integral; así mismo, ofrece soluciones efectivas y de mayor impacto que mejoran la competitividad de la misma. Además de las relaciones y correlaciones entre los miembros que integran la cadena, en búsqueda de una competitividad sistémica.

Como base para la conceptualización y el análisis de sector helicícola, para organizar la cadena productiva del caracol en Colombia, se utilizó el enfoque sistémico empleado en el proceso de planificación estratégica de la Empresa Brasileña de Investigación Agropecuaria Embrapa (Modelo 1) (Castro, 1995). Por su parte, entre los actores fuera de las unidades productivas finca, se encuentran los proveedores de insumos, las agroindustrias, las estructuras de comercialización, los consumidores y las estructuras de apoyo a la producción.

Es así como la visión de la cadena productiva debe tener tres variables fundamentales:

La producción, que incluye aspectos tales como: la planeación y organización de los factores de producción, el acceso a insumos y recursos, la producción misma, la clasificación y selección de los neonatos y caracoles de engorde, así como el envío del producto a los intermediarios. Posteriormente, se realiza el acopio, selección, empaque, transformación, diferenciación del producto, agrega valor, transporte y traspaso del producto.

En relación al mercadeo y comercialización internacional de una cadena productiva del caracol, su perspectiva se enmarca en la promoción y publicidad, el contacto de compradores, la gestión de mercado, la negociación de márgenes de precio y las condiciones de venta y, finalmente, entrega del producto.

Una de las barreras de entrada para la creación de la cadena productiva de caracol es la rentabilidad, ya que requiere que, en toda la cadena desde el productor hasta el distribuidor en el exterior, se generen beneficios.

Entre los elementos claves para organizar las funciones de los diferentes actores de la cadena, se encuentran: la normativa, en aspectos como la fijación de estándares de calidad para proveedores, la frecuencia de entregas y la calidad; lo judicial, en el control a los estándares en cumplimiento con la calidad y lo ejecutivo, en 


\section{AMBIENTE INSTITUCIONAL}

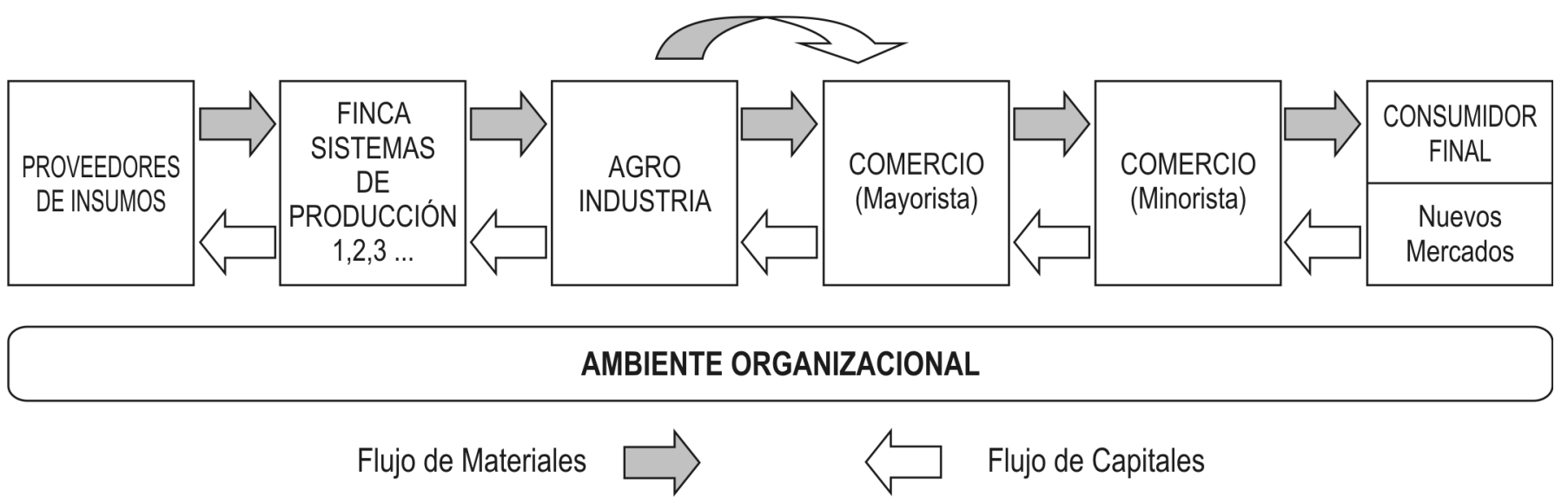

Modelo 1. Cadena productiva básica formulada por EMBRAPA, Brasil. Fuente: Castro (1995).

la coordinación de la cadena productiva, ayudar a proveedores para cumplir con los estándares y, asociación de productores para apoyar a miembros para lograr estándares.

La propuesta de la cadena productiva del caracol es un enfoque de demanda externa, haciendo referencia a la relación de los grandes minoristas, intermediarios y productores, que manejan marcas y quienes juegan el papel de coordinadores organizados en redes de comercialización.

\section{La comercialización de caracoles hacia La Unión} Europea y hacia España: Dado que en España no existe una normativa específica sobre caracoles, la cría, la producción y la comercialización se somete a las normas generales sobre sanidad animal, explotaciones ganaderas y consumo.

Es de considerar, también, que el creciente consumo mundial de caracol, como alimento, esta determinado en aproximadamente 300.000t/año, con una demanda estimada insatisfecha de 30.000t/año, cuyo precio promedio por kilo oscila entre US\$5/US\$10 y $6 € / 11 €$ y que representa una oportunidad de negocio para los empresarios del subsector helicícola colombiano.

La cantidad mínima aceptada por un importador suele ser un pallet, 700 a $1000 \mathrm{~kg}$, generalmente, es enviado de prueba esperando pedidos de mayor volumen. Los restaurantes suelen importar por sus propios medios, pero en menores cantidades, que pueden bajar hasta $500 \mathrm{~kg}$, situación que no sería rentable. El caracol vivo se exporta por avión, pero el flete es más caro. Vivo refrigerado, precocido, congelado o elaborado, se transporta en barco y el flete es más económico. "Caracoles, excepto los de mar", vivos, frescos, refrigerados, congelados, salados, secos o en salmuera, con la siguiente apertura: su nomenclatura arancelaria incluye a los caracoles dentro de la posición N.C.E. 0307.60.00.100. P: "Caracoles de tierra (H. aspersa)" y 0307.60.00.900. H: "Los demás". Los caracoles procesados y en conserva están incluidos en la posición arancelaria N.C.E 16.05.90.00 y sus caparazones en N.C.E. 05.08.00.00.

Para la comercialización, se deben considerar las regulaciones y las exigencias del mercado europeo, entre las más importantes, se encuentran: la norma HACCP (Hazard Analysis and Critical Control Points), que enfatiza en el análisis de peligros, regula la seguridad y la higiene para productos alimenticios, durante todo su ciclo y la Globalgap, que es el código de buenas prácticas agrarias de la Asociación Europea de Minoristas. El Reglamento (CEE) no 2658/87, del Consejo de 23 de julio de 1987, relativo a la nomenclatura arancelaria, estadística y al arancel aduanero común, creó una nomenclatura de mercancías; para el caso de los caracoles, el Código es 03.07.60, excepto los de mar, aptos para alimentación humana. 
El importador es el responsable de la comercialización comunitaria del producto que importe y, como tal, debe conservar, a disposición de las autoridades de vigilancia, el expediente técnico y la declaración de conformidad del fabricante. Además, en la etiqueta puede constar el código de barras, aunque no es algo que sea obligatorio, colocar en la etiqueta de presentación del producto. La AECOC (Asociación Española de Codificación Comercial) es el representante en España de EAN internacional.

Las importaciones de caracoles a la UE precisan de un certificado sanitario que otorgan aquellos organismos o autoridades certificados, según la normativa comunitaria.
La importación de caracol, se realiza en vivo, congelado, semielaborado o elaborado. Por lo que respecta a Europa, los exportadores son conscientes que la mayoría de sus clientes solicitan, por lo general, el producto vivo, especialmente, desde noviembre a marzo. Usualmente, el transporte se efectúa por vía aérea para embarques desde latinoamérica.

De lo anterior, se deduce que existe una brecha de mercado entre la producción y la demanda que requiere un suministro continuo, en el cual, Colombia bien podría abastecer si existiera un encadenamiento productivo, como se muestra en la propuesta del Modelo 2.

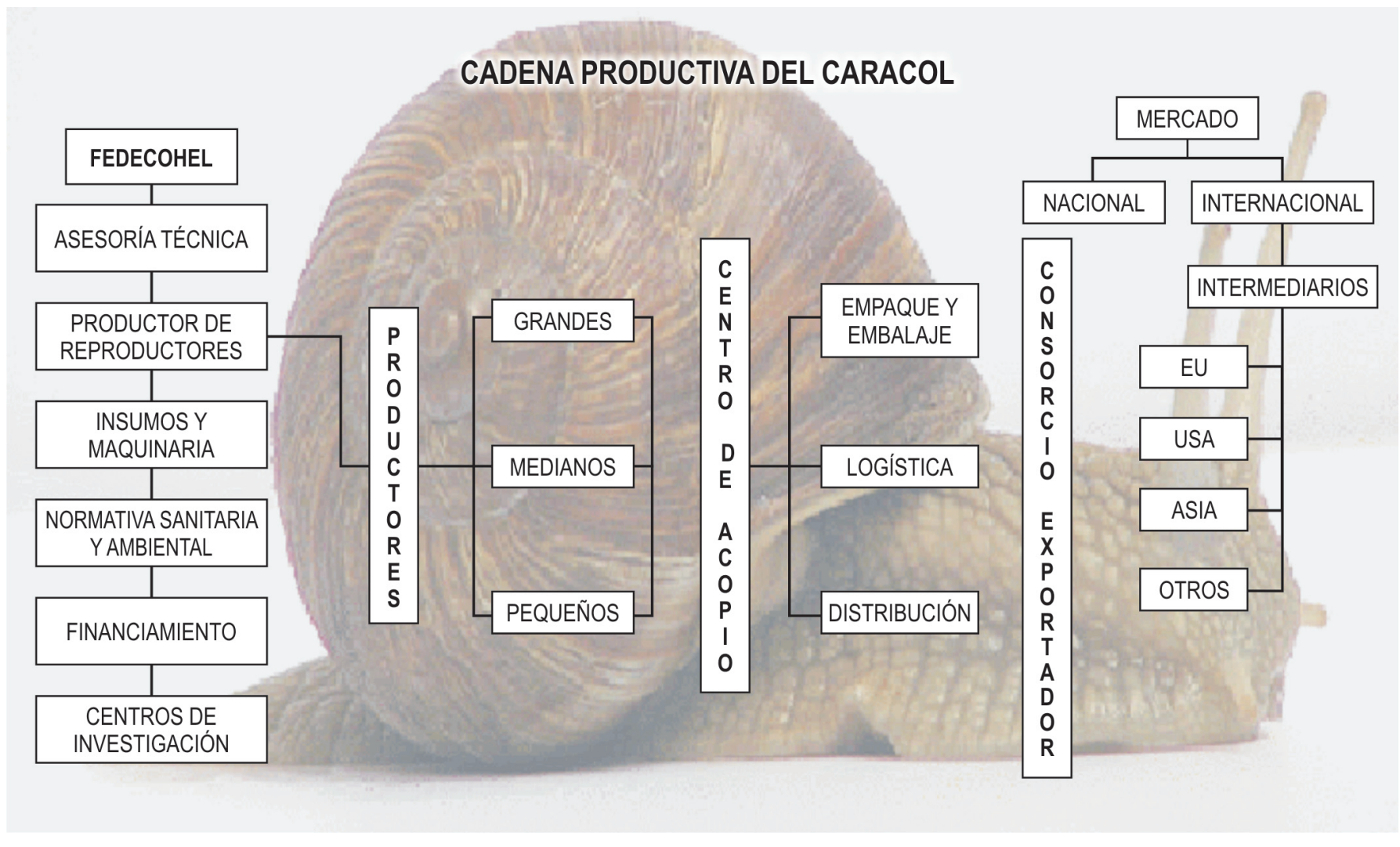

Modelo 2: Propuesta cadena productiva caracol de tierra Helix aspersa para exportación.

Cabe destacar la necesidad de crear la cadena productiva definida por la Ley 811 de 2003, como el "conjunto de actividades que se articulan técnica y económicamente desde el inicio de la producción y elaboración de un producto agropecuarios, hasta su comercialización final. La Cadena puede ser conformada de común acuerdo, a nivel nacional, a nivel de una zona o región productora, por los productores, empresarios, gremios y organizaciones más representativos tanto de la producción agrícola, pecuaria, forestal, acuícola, pesquera, como de la transformación, la comercialización, la distribución, y de los proveedores de servicios e insumos de tal manera que se manejen propuestas unificadas y se puedan aprender entre los mismos helicicultores de sus experiencias". 
Con la cadena, se tendrá la oportunidad de confrontar los diferentes sistemas de cría estudiados, sus ventajas y desventajas comparativas, en cuanto a producción, comercialización y manejo ambiental. Se establecen los instrumentos requeridos para aplicar en los casos particulares e incluye estrategias que permitan acercar más a los helicicultores, como Formación de multiplicadores y Sistema de referenciación Helicícola.

Normatividad para la comercialización de carne de caracol en Colombia: Mientras en el mercado español de caracoles la norma aplicada es general, en el mercado colombiano, ya existe una norma específica para poder exportar la carne de caracol y los derivados de la helicicultura. Antes de la existencia de la norma, en Colombia se producía el caracol en condiciones marginales y pequeñas cantidades, pero la existencia y puesta en práctica de la norma, ha posibilitado el poder mejorar las cantidades de producción y la calidad en la comercialización.

La Ley colombiana 1011 de 2006, reglamentada por el decreto 2490, del 9 de julio de 2008, decreta lo relacionado con la recolección, el cultivo, la selección, el manejo, el transporte, la comercialización y la exportación y otras disposiciones de la especie $H$. aspersa, como objeto regulador y determinante de las normas vigentes en materia de alimentos, calidad del agua, insumos agrícolas y pecuarios, protección al medio ambiente y los recursos naturales, ingredientes utilizados en la industria de alimentos, desechos de producción, comercialización, certificación y etiquetado

De otra parte, las definiciones Caracol, Ciclo Cerrado, Género Helix, Helix aspersa, modalidad o manejo Intensivo, operador, Plan de manejo Ambiental entre otras, permiten la correcta aplicación de lo dispuesto en el decreto en cuanto al reglamento técnico, por el cual, se señalan los requisitos sanitarios que deben cumplir los establecimientos dedicados al procesamiento, envase, expendio, transporte, Importación, exportación y comercialización del caracol.

La producción de la especie $H$. aspersa en cualquiera de sus modalidades, está supeditada a lo que se disponga en el Plan de Manejo Ambiental del zoocriadero. Las actividades de tipo comercial, solamente se podrán realizar una vez se encuentre autorizada la fase comercial por parte de la Corporación Autónoma Regional o de Desarrollo
Sostenible competente y bajo los términos y condiciones allí establecidas, además, deben estar amparadas por un Sistema de Administración Ambiental (S.A.A).

El salvoconducto de movilización, así como su procedencia y destino, se determinan conforme lo dispuesto en la Resolución 0438 de 2001, expedida por el Ministerio de Ambiente, Vivienda y Desarrollo Territorial o por la norma que la modifique o sustituya y las medidas de bioseguridad que establezcan las autoridades ambientales y sanitarias, o amparados por el registro INVIMA.

Así mismo, los productores y/o exportadores deberán cumplir con los trámites y los requisitos establecidos para la expedición del certificado zoosanitario de exportación que expide el ICA (Instituto Colombiano Agropecuario), dando cumplimiento a las normas establecidas por la autoridad sanitaria de destino y con los permisos que, para el efecto, corresponde expedir al Ministerio de Ambiente, Vivienda y Desarrollo Territorial, conforme lo dispone la Resolución 1367 de 2000, del Ministerio del Medio Ambiente y demás requisitos que se establecen en las normas aplicables.

Cabe resaltar que la Organización Mundial de Comercio fue notificada de la existencia de las normas para comercialización de caracoles, mediante los documentos identificados con la nomenclatura G/TBT/N/COL/128.

\section{CONCLUSIONES Y RECOMENDACIONES}

La cría sostenible de caracoles en Colombia podría reducir el impacto ambiental causado por una especie no nativa, si se fomenta y permite su comercialización y el aprovechamiento de sus productos y subproductos, lo cual, podría generar ingresos y convertirse en una fuente de empleo, aprovechando racionalmente, las especies ya establecidas en el país.

La capacitación, la asesoría y el acompañamiento en aspectos relacionados con la producción y la comercialización del caracol permitieran ser más competitivo al sector helícicola colombiano; como se trata de una actividad incipiente y nueva, es necesario desarrollar su cadena productiva y fomentar campañas 
de concientización en la comunidad, encaminadas a mejorar aspectos productivos, de comercialización, de asociatividad y de cooperativismo.

Para fortalecer la actividad helicícola en el país, se requiere crear sinergias que permitan diseñar y proponer la cadena productiva del caracol de tierra, de manera que posibilite desarrollar la especialización de los componentes de la cadena productiva (materias primas, pie de cría, levante, procesamiento, comercialización, la trazabilidad, entre otros factores), para mejorar la competitividad, con el fin de desarrollar exportaciones hacia el mercado europeo e identificar las características de la demanda, particularmente del consumidor de caracol, como alimento en España.

Con la existencia de una norma específica en Colombia, se crea la apertura de nuevos mercados no solo hacia España y Francia, consumidores más destacados, sino hacia otros mercados, como los de oriente, Norte América, Canadá y de América Latina. Además, con la norma se protege el ambiente y se diversifica la producción y comercialización agropecuaria.

La participación en ferias internacionales del sector alimenticio le ha permitido conocer a los helicicultores la competencia internacional, así como los gustos y las preferencias de los consumidores para tener una visión global del sector y, de esta forma, desarrollar estrategias que le den bases para ser competitivos en los mercados internacionales, pues existen oportunidades reales y potenciales por aprovechar.

El desarrollo de la helicicultura debe estar orientado a crear una cultura de su consumo a nivel nacional, hasta lograr niveles de producción, que permitan el ingreso al mercado externo, impulsado por el enorme consumo de caracoles terrestres, para la gastronomía en el mundo entero; alrededor de la mitad de los caracoles comercializados en la UE tienen como destino la industria del congelado y las conservas, lo que requiere más investigación y desarrollo.

Es necesario mejorar el nivel productivo de zoocriaderos de caracol establecidos en el departamento de Cundinamarca porque es bajo o incipiente, como se observa en la tabla 1.

Se requiere mejorar la cadena logística del sector, llevando registros sistematizados de la edad del animal, así como el control a proveedores del alimento utilizado, la calidad del agua, los insumos y los materiales utilizados y, así, aumentar la calidad del producto final.

Tabla 1. Resultados, productividad y comercialización, basado en la encuesta.

\begin{tabular}{|c|c|c|c|c|c|}
\hline Sistema & $\begin{array}{c}\text { Manejo } \\
\text { Zoocriadero }\end{array}$ & $\%$ Manejo & $\begin{array}{c}\text { Manejo } \\
\text { Ambiental }\end{array}$ & $\begin{array}{c}\text { Producción real } \\
\text { o estimación } \\
\text { kg/mes }\end{array}$ & Comercialización \\
\hline $\begin{array}{c}\text { Intensivo } \\
\text { (Municipio de Funza) }\end{array}$ & Convencional & 14,1 & Bajo & 300 en pie estimada & Incipiente \\
\hline $\begin{array}{c}\text { Intensivo } \\
\text { (Región del Tequendama) }\end{array}$ & Mixto & 14,4 & Bueno & 50 & Incipiente \\
\hline $\begin{array}{c}\text { Intensivo-industria } \\
\text { (Municipio de Chía) }\end{array}$ & Mixto & $14 .$, & Medio & 20 (Estimación) & Incipiente \\
\hline $\begin{array}{c}\text { Mixto } \\
\text { (Municipio de Cajicá) }\end{array}$ & Mixto & 14,1 & Bajo & 140 (Estimación) & Bajo \\
\hline $\begin{array}{c}\text { Extensivo } \\
\text { (Región del Guavio) }\end{array}$ & Orgánico & 14,3 & Medio & $30-60$ & Medio \\
\hline $\begin{array}{c}\text { Intensivo } \\
\text { (Municipio de Choachí) }\end{array}$ & Orgánico & 14,3 & Medio & 5.000 & Bajo \\
\hline $\begin{array}{c}\text { Intensivo- industria } \\
\text { (Región del Sumapaz) }\end{array}$ & Mixto & 14,4 & Bueno & $\begin{array}{c}2500 \\
(\text { Estimación) }\end{array}$ \\
\hline
\end{tabular}


Es importante concientizar a los helicicultores del trabajo en equipo para que sirvan en cada región, con el apoyo y participación de la academia, el gobierno, el gremio y los helicicultores independientes, con quienes se intercambien experiencias y se implementen acciones para optimizar procesos, mejorar su desempeño ambiental y aumentar la productividad.

Se recomienda buscar la implementación de una herramienta de mejoramiento "Sistema de Referenciación para el sector helicícola", con la cual, más que medir, comparar y establecer un nivel de desempeño entre diferentes empresas, aprender de otros a través de la identificación continua y comprensión de las mejores prácticas para desarrollar un determinado proceso o actividad. Finalmente, se sugiere acogerse a la iniciativa de producir orgánicamente, trabajando en trazabilidad, en análisis de ciclo de vida y en sostenibilidad ambiental y productiva.

\section{BIBLIOGRAFÍA}

CASTRO, A. 1995. Pesquisa Agropecuaria Brasileira. Brasil Emprapa. 533p.

CORPORACIÓN FINANCIERA NACIONAL. 2003 El cultivo de escargot. Ed Proexant. Santiago de Chile, 176p.

GARCÍA, I. 2005. Estudio de prefactibilidad de la cría del Escargot en Colombia (Trabajo de grado) Bogotá. Fundación Universitaria San Martín. Escuela de Finanzas. 113p.

GIRALDO, P.; RESTREPO, Q.; WAHANIK, P. 2006. Helicicultura colombiana: ¿Alternativa productiva y exportadora? 18p.

GUTIÉRREZ, F. 2002. El impacto de las especies introducidas en Colombia. Dirección de Ecosistemas del Ministerio de Ambiente, Vivienda y Desarrollo Territorial. 126p.

HOBBS, R.; CRAMER, V. 2000. Cadenas productivas de agricultura campesina. Coordinadora Integración y Organizaciones Económicas. Bolivia. 42p.
LEY 99 de 1993. Prohibición a la importación de Helix aspersa.

LEY 165 de 1994, por la cual se aprobó el Convenio Sobre la Diversidad Biológica.

LEY 811 de 2003. Congreso de Colombia (junio 26). Diario Oficial No. 45.236 de 2 de julio de 2003.

LEY 1011. Congreso de Colombia 2006. Diario Oficial No. 46.160 de 23 de enero de 2006.

LEY 24 de 2008. Congreso de Colombia. Por la cual se autoriza la producción y comercialización de caracol.

MONTES, E.A.; MONTES, F. 2002 Exportaciones de caracoles. Rev. Quipukamayoc Segundo Semestre. Lima, p.47.

RESOLUCIÓN 0438 de 2001. Ministerio de Medio Ambiente. Por la cual se estableció el salvoconducto único para la movilización de especímenes biológicos.

RESOLUCIÓN 1367 del 2000. Ministerio del medio Ambiente. Por la cual se establece el procedimiento para las autorizaciones de importación y exportaciones de especimenes de la diversidad biológica.

REUNA, RED DE EXTENSIÓN ACADÉMICA DE LA UNIVERSIDAD NACIONAL,. 2005. Validación de un sistema productivo con Helix aspersa bajo las condiciones agroecológicas del altiplano de Popayán. 26p.

RODRIGUES, M.P. 1991. Manual práctico para a criação de caracóis (escargots). 2.ed. São Paulo: Ícone, 123p.

ROUSSELET, M. 1986. Cria de caracol. Madrid. Mundi Prensa, 144p.

SÁNCHEZ, N. 2004. El caracol Helix aspersa: Biología, Cultivo, Manejo, producción y comercialización. 100p.

Recibido: Agosto 25 de 2008

Aceptado: Abril 27 de 2009 